\title{
A UNIFORM BOUNDEDNESS THEOREM ${ }^{1}$
}

JOHN W. BRACE AND ROBERT M. NIELSEN

A variation on the uniform boundedness theorem [2, p. 105] of functional analysis is obtained by replacing pointwise boundedness by a slightly stronger type of boundedness. This makes it possible to remove the category requirements from the set over which it is desired to have a collection of functions uniformly bounded. The concept of boundedness to be utilized is called boundedness on Cauchy sequences (see Definition 1). When a Cauchy sequence converges in the domain of a family of continuous functions, boundedness on a Cauchy sequence is equivalent to pointwise boundedness of the family at the limit point of the point of the sequence. When the domain of the continuous functions is a metric space, boundedness on the collection on all Cauchy sequences is equivalent to pointwise boundedness of the continuous extensions on the completion of the domain. The interesting case is when the domain is not metrizable because boundedness on Cauchy sequences does not necessarily imply pointwise boundedness of the extended functions on the completion or even on the sequential completion of the domain.

The main result of the paper is given in Theorem 1 which is followed by six corollaries. The sixth corollary is especially interesting in stating that the closure of an infrabarrelled space in its second adjoint space is a barreled space.

The theorem on uniform boundedness immediately suggests an absorption theorem which is Theorem 2 of the paper. Theorem 1 does not follow as a corollary of Theorem 2 as is the case of the classical uniform boundedness theorem [2, p. 105].

Definition 1. A family $H$ of continuous linear transformations with common domain a linear topological space $E$ and ranges in a locally convex space $F$ is bounded on a Cauchy sequence $\left\{x_{n}\right\}$ in $E$ if for every neighborhood $V$ of the origin in $F$ there exists a positive real number $r$ with the property that for each $f$ in $H$ there is an $N_{f}$ such that $f\left(x_{n}\right)$ is in $r V$ for all $n>N_{f}$.

The following theorem is stated in the context of the definition.

Presented to the Society, November 24, 1965; received by the editors March 10 , 1966.

1 This research was supported in part by the National Science Foundation and the University of Delaware Research Foundation. 
THEOREM 1. The family $H$ of continuous linear transformations is uniformly bounded on a bounded convex subset $B$ of $E$ if and only if $H$ is bounded on every Cauchy sequence in $B$.

Proof. In the completion $\hat{E}$ of $E$ let $M$ be the linear span of the closed convex circled hull of $B$. Place on $M$ the pseudonorm obtained taking the unit ball to be the closed convex circled hull of $B$. Since the set $B$ is convex and bounded, it can be shown that the closed convex circled hull of $B$ is bounded even when the topology on $E$ is not locally convex. Thus the resulting pseudonorm topology is stronger than the original topology. Noting that the unit ball for the pseudonorm is closed for the original topology in which $M$ is complete, it follows that $M$ is complete for the pseudonorm [2, p. 91, last part of the proof of 10.3].

Since a Cauchy sequence in $B$ for the pseudonorm is Cauchy for the original topology, without loss of generality it can be assumed that $\hat{E}$ is a complete pseudometrizable space and all members of $H$ have been continuously extended to $\hat{E}$ with their ranges now in the completion $\hat{F}$ of $F$. The family $H$ is pointwise bounded on $\bar{B}$ because it is bounded on Cauchy sequences of $B$. If $V$ is an arbitrary convex circled closed neighborhood of 0 in $F$, then $U=\bigcap\left\{f^{-1}(V): f \in H\right\}$ absorbs each point of $\bar{B}$, which is of the second category in itself. Therefore by 10.1 of [2], $U$ absorbs $\bar{B}$ and thus $H$ is uniformly bounded on $B$. The conclusion remains valid when the members of $H$ are again restricted to $E$ with their ranges in $F$. The converse is immediate.

Corollary 1. If $A$ is a bounded subset of $E$ having a dense convex subset $B$ such that every Cauchy sequence in $B$ converges to a point $A$, then a family $H$ of continuous linear transformations will be uniformly bounded on $A$ if and only if $H$ is pointwise bounded on $A$.

Proof. Since uniform boundedness of $B$ implies uniform boundedness on $A$, and pointwise boundedness of $A$ implies boundedness on all Cauchy sequences in $B$, the corollary is obtained by applying the theorem to the set $B$. Note that $A$ need not be sequentially complete.

When the space $E$ is infrabarrelled the strongly bounded subsets of the adjoint space are equicontinuous. Combining this fact with Theorem 1 gives the following corollary

Corollary 2. If $E$ is infrabarrelled and $H$ is a collection of continuous linear functionals such that $H$ is bounded on all Cauchy sequences in $E$ then $H$ is equicontinuous. 
Combining Theorem 1 and Corollary 2 we obtain the following corollary, which generalizes 20.4 (iv) of [2].

Corollary 3. Let $D$ be a dense subspace of $E$ such that each Cauchy sequence in $D$ converges to a point in $E$. If $D$ is infrabarrelled, then $E$ is barrelled.

For the bounded extension of a locally convex topology there is the following corollary [2, p. 183].

Corollary 4. A collection $G$ of bounded linear transformations with common domain the locally convex space $E$ and range in the locally convex space $F$ is equicontinuous for the bounded extension of the topology on $E$ if and only if $G$ is bounded on all Cauchy sequences of $E$ for the original topology.

In the following corollary the space $\tilde{E}$ is the smallest locally convex space in which $E$ can be embedded such that every Cauchy sequence in $E$ converges. A representation of $\tilde{E}$ is given in [1].

Corollary 5. A subset of the adjoint space $E^{*}$ is strongly bounded if and only if it is weak $\left(E^{*}, \tilde{E}\right)$-bounded.

In the above corollary weak $\left(E^{*}, \tilde{E}\right)$-boundedness implies boundedness on all Cauchy sequences of $E$ making Theorem 1 applicable. Reflecting on the proof of the theorem relative to the corollary, it is not clear that $\tilde{E}$ should be the smallest place that will give the desired result.

CoRollary 6. If $E$ is infrabarrelled then the strong closure of $E$ in $E^{* *}$ is a barrelled space.

Observe that each Cauchy sequence in $E$ converges to a point in $E \subset E^{* *}$ and apply Corollary 3.

The above ideas are now applied to obtain an absorption theorem. The usual absorption of points is replaced by absorption of Cauchy sequences (see Definition below).

Definition 2. In linear topological space $E$ a subset $A$ absorbs a Cauchy sequence $\left\{x_{n}\right\}$ if there is a positive real number $r$ such that for every real number $s>r$ and positive integer $N$ there exists an $n>N$ with the property that $x_{n}$ is in $s A$.

The definition is created in order that the closure of $A$ in the completion of $E$ will absorb the limit point of a Cauchy sequence if it absorbs the Cauchy sequence.

Theorem 2. Let $A$ be a closed convex subset and $B$ a sequentially 
closed convex and bounded subset of a linear topological space E. If $A$ absorbs (i) every point of $B \cup-B$ and (ii) every nonconvergent Cauchy sequence in $B$, then $A$ absorbs $B$.

Proof. As in the proof of the first theorem, let $M$ be the complete pseudonormed space having as its unit ball the closed circled convex hull of $B$ in the completion $\hat{E}$ of $E$. The closure $\bar{B}$ of $B$ in $M$ is of the second category in itself. The hypothesis was designed in order that the closure $\bar{A}$ of $A$ in $\hat{E}$ absorbs every point of $\bar{B}$.

Applying 10.1 of [2] to $M$ (with the pseudonorm topology), one sees that $\bar{A} \cap M$ absorbs $\bar{B}$ (pseudonorm closure); hence $\bar{A}$ absorbs $\bar{B}$, and finally $A$ absorbs $B$ since $A$ is closed in $E$.

The authors gratefully acknowledge the referee's helpful suggestions and clarifications of several points; in particular Corollary 3 of Theorem 1 was pointed out by him.

\section{BiBLIOGRAPHY}

1. J. W. Brace and R. M. Nielsen, Completions, Math. Ann. (to appear).

2. J. L. Kelley and I. Namioka, Linear topological spaces, Van Nostrand, Princeton, N. J., 1963.

UNIVERSITY OF MARYLAND AND

UNIVERSITY OF DELAWARE 